\title{
Nano/Microstructured Materials: Rapid, Low-Cost, and Eco-Friendly Synthesis Methods
}

\author{
Amir Kajbafvala, ${ }^{1}$ Minghang Li, ${ }^{2}$ Hamed Bahmanpour, ${ }^{3}$ Mohammad H. Maneshian, ${ }^{2}$ and \\ Alexander Kauffmann ${ }^{4}$ \\ ${ }^{1}$ Department of Materials Science and Engineering, North Carolina State University, 911 Partners Way, Engineering Building I, \\ Raleigh, NC 27695-7907, USA \\ ${ }^{2}$ Department of Materials Science and Engineering, University of North Texas, Denton, TX 76203, USA \\ ${ }^{3}$ Department of Chemical Engineering and Materials Science, University of California, Davis, CA 95616, USA \\ ${ }^{4}$ Leibniz Institute for Solid State and Materials Research Dresden, P.O. Box 27 01 16, 01171 Dresden, Germany \\ Correspondence should be addressed to Amir Kajbafvala; akajbaf@ncsu.edu
}

Received 9 July 2013; Accepted 9 July 2013

Copyright (C) 2013 Amir Kajbafvala et al. This is an open access article distributed under the Creative Commons Attribution License, which permits unrestricted use, distribution, and reproduction in any medium, provided the original work is properly cited.

Chemists are required to generate a diverse array of organic and inorganic molecules using eco-friendly conditions, including low-cost and short-reaction times. A primary driver of synthetic chemistry is, therefore, the development of efficient and environmentally benign synthetic protocols, as the pressure to produce the myriad of substances required by society in an eco-friendly fashion has continued to increase. Up to now, many works have been published on synthesis and characterization of various multidimensional micro/nanostructured materials via diverse synthesis methods. However, complex conditions and long synthesis time were required for most of these technologies.

Microwave-assisted processing methods, as good examples, have been developed for a variety of applications in organic and inorganic synthesis and transformations. There are many examples of the successful application of MW-assisted green chemistry for synthesis of micro/nanostructured materials in the literature. This special issue addresses rapid methods for synthesis of organic and inorganic micro/nanomaterials to maximize the efficient use of safer raw materials and to reduce waste for fabrication of various micro/nanostructured materials. This special issue contains nineteen papers including seventeen research and two review papers.

In "Studying the effects of adding silica sand nanoparticles on epoxy based composites," T. Ahmad et al. produced
Epoxy- $\mathrm{SiO}_{2}$ nanocomposites using Tronoh silica sand nanoparticles as reinforcement. They reported that density of the epoxy- $\mathrm{SiO}_{2}$ nanocomposites increases from $1.157 \mathrm{~g} / \mathrm{cm}^{3}$ to $1.244 \mathrm{~g} / \mathrm{cm}^{3}$. The mechanical properties of the fabricated nanocomposites decrease with the addition of silica sand nanoparticles due to improper mixing of the nanoparticles. SEM micrographs show white and agglomerate zones on fracture surfaces which indicate that silica sand nanoparticles are not homogeneously dispersed in epoxy.

In their paper, J. Panda et al. studied a "Green chemistry approach for efficient synthesis of Schiff bases of Isatin derivatives and evaluation of their antibacterial activities." They synthesized Schiff base of Isatin derivatives by conventional and microwave irradiation method. With microwave synthesis, the yield of product increases from $60 \%$ up to $85 \%$ as compared to conventional method. By microwave irradiation the reactions are completed within 5-10 min, which reduced the time, waste, and formation of byproduct. This microwave-assisted synthesis is reported as a simple and eco-friendly route. From their results of antibacterial studies, they concluded that compounds exhibited significant antibacterial activities against both Gram-positive and gramnegative organisms.

In his paper, A. K. Singh presented "Microwave assisted growth of ZnO nanorods and nanopolypods nanostructure thin films for gas and explosives sensing." It was shown that use 
of triethanolamine (TEA) as capping agent has resulted in the additional growth of $\mathrm{ZnO}$ NPPs. The structural analysis shows hexagonal wurtzite structure of $\mathrm{ZnO}$ with planes highly oriented along (002) direction. The sensing study of $\mathrm{ZnO} \mathrm{NRs}$ and $\mathrm{ZnO}$ NRs/NPPs samples shows that the sensor response $(S)$ increases with temperature for both liquefied petroleum gas (LPG) and oxygen, while, with the increase in gas concentration from 0.2 to $0.4 \mathrm{vol} \%$, the sensor response increases gradually and attains saturation for LPG, and, for oxygen, the response is found to be linear with the increase in gas concentration. The sensor sensing and the recovery time analysis show that the recovery time reduces with the increase in operation temperature for both gas and explosives. Additionally, the recovery time achieved for LPG at 0.4 vol\% concentration is much lesser than that previously reported for $\mathrm{ZnO}$ thin film with much higher LPG concentration.

In their paper, "Synthesis of $\mathrm{ZnO}$ nanostructures by microwave irradiation using albumen as a template," $\mathrm{T}$. Prakash et al. synthesized $\mathrm{ZnO}$ nanostructures via a microwave irradiation method in the presence of albumen as a biotemplate. The composition and morphology of the nanostructures are affected by the presence of albumen as a template. The optical band gap of the polycrystalline $\mathrm{ZnO}$ nanoparticles increases from 3.22 to $3.25 \mathrm{eV}$ for the sample synthesized in the presence of albumen because of the defects' decrease. The good quality of $\mathrm{ZnO}$ nanostructures produced was also demonstrated by PL studies.

In "Rapid green synthetic protocol for novel trimetallic nanoparticles," B. Karthikeyan and B. Loganathan presented the preparation of nanosized trimetallic nanocomposites and trimetallic nanotubes from an aqueous solution of corresponding metallic precursors. They reported a simple microwave irradiated synthesis of Au-Pt-Ag trimetallic nanocomposites and $\mathrm{Au}-\mathrm{Pd}-\mathrm{Pt}$ trimetallic nanotubes. EDX analysis confirmed that the percentage of $\mathrm{Pt}$ is maximum on the surface of trimetallic nanoparticles and nanotubes.

In their paper, "Photocatalytic properties of microwavesynthesized $\mathrm{TiO}_{2}$ and $\mathrm{ZnO}$ nanoparticles using malachite green dye," A. K. Singh and U. T. Nakate synthesized $\mathrm{TiO}_{2}$ and $\mathrm{ZnO}$ nanoparticles using a green, efficient, and costeffective microwave method. The photocatalytic activity of these nanoparticles was studied for malachite green (MG) dye under UV light. The photocatalytic tests showed that $\mathrm{TiO}_{2}$ and $\mathrm{ZnO}$ nanoparticles have $49.35 \%$ and $23.31 \%$ photodegradation efficiency, respectively, for $\mathrm{MG}$ dye under UV light. $\mathrm{TiO}_{2}$ nanoparticles show more than two-order photodegradation property for $\mathrm{MG}$ dye as compared to $\mathrm{ZnO}$.

In "Tuning the pore size in ionic nanoparticle networks" M. -A. N. Gauthey et al. described a new ionic nanoparticle networks (INNS) material based on zirconia nanoparticles and compared the porous characteristics of different INNs, with various metal oxide nanoparticles. In these materials, the liking imidazolium moieties are maintained in the network and thus remained accessible to adsorbed molecules. The INN based on zirconia nanoparticles linked by ionic liquidlike imidazolium bridging units. It was shown that the porous characteristics of the INN depend on the size and shape of the nanoparticles. INNs based on large spherical nanoparticles, like $\mathrm{SiO}_{2}$, are macroporous, while INNs based on small spherical nanoparticles, like $\mathrm{TiO}_{2}$, are microporous.

In their paper, Y. -W. Chen and D. S. Lee investigated "Selective hydrogenation of p-chloronitrobenzene on nanosized $p d N i B$ catalysts." They prepared a series of $\mathrm{PdNiB}$ nanoalloy catalysts with various $\mathrm{Pd}$ contents by chemical reduction method with $\mathrm{NaBH}_{4}$ as the reducing agent. The magnetization of $\mathrm{PdNiB}$ remarkably increased with doping $\mathrm{Pd}$ into NiB. High activity and selectivity on $\mathrm{PdNiB}$ in the hydrogenation of $p$-CNB could be attributed to both ensemble effect and electronic effect.

In their paper, "Microwave-assisted synthesis of mixed metal-oxide nanoparticles," A. Verma et al. synthesized $\mathrm{ZrO}_{2}$ and $\mathrm{ZrTiO}_{4}$ nanoparticles by microwave-assisted citrate solgel method. Nanoparticles of $\mathrm{V}_{2} \mathrm{O}_{5}$ supported on $\mathrm{ZrO}_{2}$ were also synthesized by microwave-assisted solution combustion method. The low-angle powder XRD measurements confirm the mesoporous nature of $\mathrm{ZrV}_{2} \mathrm{O}_{7}$ and formation of single phase material up to $10 \mathrm{wt} \%$ of vanadium incorporation. The HRTEM data confirms the formation of single phase $t$ $\mathrm{ZrO}_{2}$. Raman spectra further support the crystalline phase as well as the specific bands to show the modes of vibration in $\mathrm{Zr}-\mathrm{O}$ system, whereas the specific bands indicate the modes of vibration in $\mathrm{Zr}-\mathrm{Ti}-\mathrm{O}$ and $\mathrm{ZrV}_{2} \mathrm{O}_{7}$ systems. The crystallite sizes were found to be in the ranges of $\sim 5-10 \mathrm{~nm}$, $\sim 2-5 \mathrm{~nm}$, and $\sim 20-50 \mathrm{~nm}$ for $\mathrm{ZrO}_{2}, \mathrm{ZrTiO}_{4}$, and $\mathrm{ZrV}_{2} \mathrm{O}_{7}$, respectively.

In their review article, "Naturally self-assembled nanosystems and their templated structures for photonic applications," K. Pradeesh et al. discussed fabrication, structural, and optical exciton features of naturally self-assembled lowdimensional IO-hybrid nanosystems. While the fabrication of these self-assembled systems is usually from solution chemistry techniques, a novel device-compatible thin film fabrication from very inexpensive method, that is, intercalation, was reviewed. Finally, the designing and fabrication of optoelectronic compatible photonic architectures from these IO-hybrids, especially from template-assisted method, have been clearly discussed.

In "Rapid, low-cost, and ecofriendly approach for iron nanoparticle synthesis using Aspergillus oryzae TFR9," J. C. Tarafdar and R. Raliya demonstrated a rapid, low-cost, and eco-friendly synthesis method for fabricating iron nanoparticles using the fungi Aspergillus oryzae TFR9.

In their paper, "CuO-CeO nanocomposite: an efficient recyclable catalyst for the synthesis of aryl-14H-dibenzo[aj]xanthenes," J. Albadi et al. developed a new, efficient, and green procedure for the synthesis of biologically important xanthene derivatives catalyzed by recyclable $\mathrm{CuO}-\mathrm{CeO}_{2}$ nanostructured catalyst, under solvent-free conditions. This catalyst can promote the yields and reaction times over eight runs without noticeable loss in its efficiency.

In their paper, A. K. Mittal et al. studied "Synthesis of gold nanoparticles using whole cells of Geotrichum candidum." Various reaction parameters like cell age, temperature, $\mathrm{pH}$, cell mass, and metal ion concentration were optimized to 
increase the yield and to improve the dispersity of nanoparticles. The gold nanoparticle throughout the cell mass suggests that $\mathrm{Au}^{+3}$ ions entered the cells through a transport system. The presence of different metabolic enzymes inside the cells or cytoplasm probably reduced $\mathrm{Au}^{+3}$ to $\mathrm{Au}^{0}$ and capped it by protective peptide/proteins.

In their article, "Electrochemical method for Ag-PEG nanoparticles synthesis," M. V. Roldán et al. presented an electrochemical method to prepare Ag nanoparticles using polyethylene glycol (PEG) as stabilizer. The PEG chain length does not show effects on the shape of the nanoparticles obtained, which are spherical for all the cases. Nevertheless, with the longer polymer chain length, it is possible to control the particle size through the PEG concentration.

In their paper, B. M. Sahoo et al. studied an "Ecofriendly and facile one-pot multicomponent synthesis of thiopyrimidines under microwave irradiation." With the help of microwave synthesis, the yield of product increased from $55 \%$ up to $85 \%$ as compared to conventional synthesis. In addition, the reaction time is reduced from $4-8 \mathrm{hrs}$ to $5-10 \mathrm{~min}$.

In their paper, V. L. Chandraboss et al. studied "Solgel synthesis of $\mathrm{TiO}_{2} / \mathrm{SiO}_{2}$ and $\mathrm{ZnO} / \mathrm{SiO}_{2}$ composite films and evaluation of their photocatalytic activity towards methyl green." In this work, $\mathrm{TiO}_{2} / \mathrm{SiO}_{2}$ and $\mathrm{ZnO} / \mathrm{SiO}_{2}$ composite films were prepared by the sol-gel method. These $\mathrm{TiO}_{2} / \mathrm{SiO}_{2}$ and $\mathrm{ZnO} / \mathrm{SiO}_{2}$ films were then used for the photodegradation of methyl green (MG) under UV-light irradiation.

In "Antibacterial activity of silver nanoparticles synthesized by bark extract of Syzygium cumini," R. Prasad and V. S. swamy used the silver nanoparticles synthesized from the bark extract of S. cumini and its antibacterial effect on the bacteria, namely, Escherichia coli, Staphylococcus aureus, Pseudomonas aeruginosa, Azotobacter chroococcum, and Bacillus licheniformis. In the present study, silver nanoparticles are synthesized at room temperature within a less span of time. The size of the nanoparticles ranges from 20 to $60 \mathrm{~nm}$ with spherical shape. These nanoparticles showed a broad spectrum antimicrobial activity against both Grampositive and Gram-negative bacteria. Investigation on the antibacterial activity of synthesized silver nanoparticles using S. cumini extract against Staphylococcus aureus and Bacillus licheniformis reveals high potential as antimicrobial agent in pharmaceutical, food, and cosmetic industries.

In their paper, "Green synthesis of nanocrystalline $\mathrm{Cu}_{2} \mathrm{ZnSnS}_{4}$ powder using hydrothermal route," S. K. Verma et al. presented a simple and relatively safe approach of hydrothermal synthesis of the quaternary semiconductor CZTS nanoparticles. Spherical nanoparticles with diameters of about 4-5 $\mathrm{nm}$ are obtained without using any expensive vacuum facilities or high temperature annealing temperature. thanks are extended to all reviewers for enhancing the quality of these papers.

Amir Kajbafvala Minghang $\mathrm{Li}$ Hamed Bahmanpour Mohammad H. Maneshian Alexander Kauffmann

\section{Acknowledgments}

We would like to express our kind appreciation to all authors in this special issue for their contribution. Furthermore, 

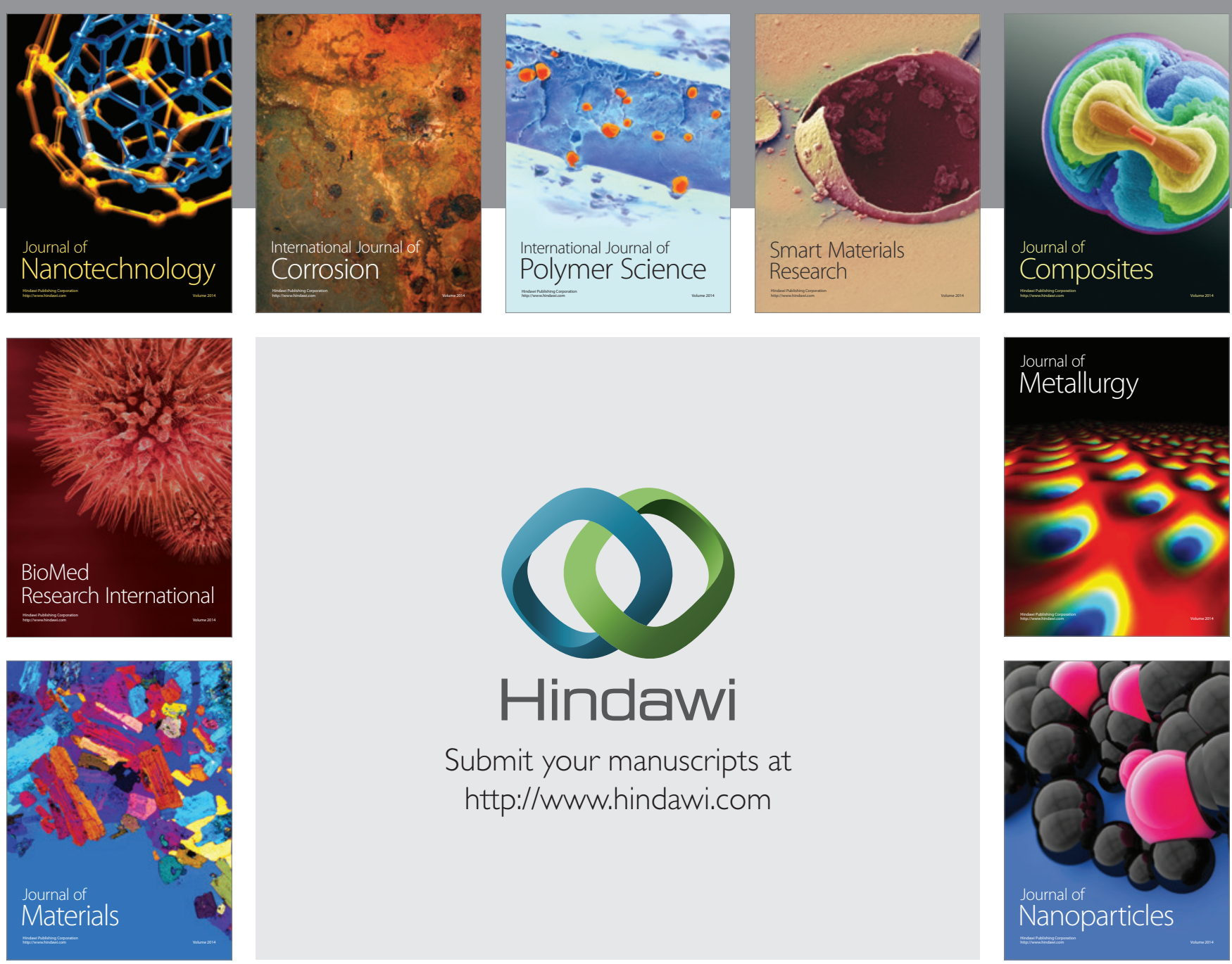

Submit your manuscripts at http://www.hindawi.com
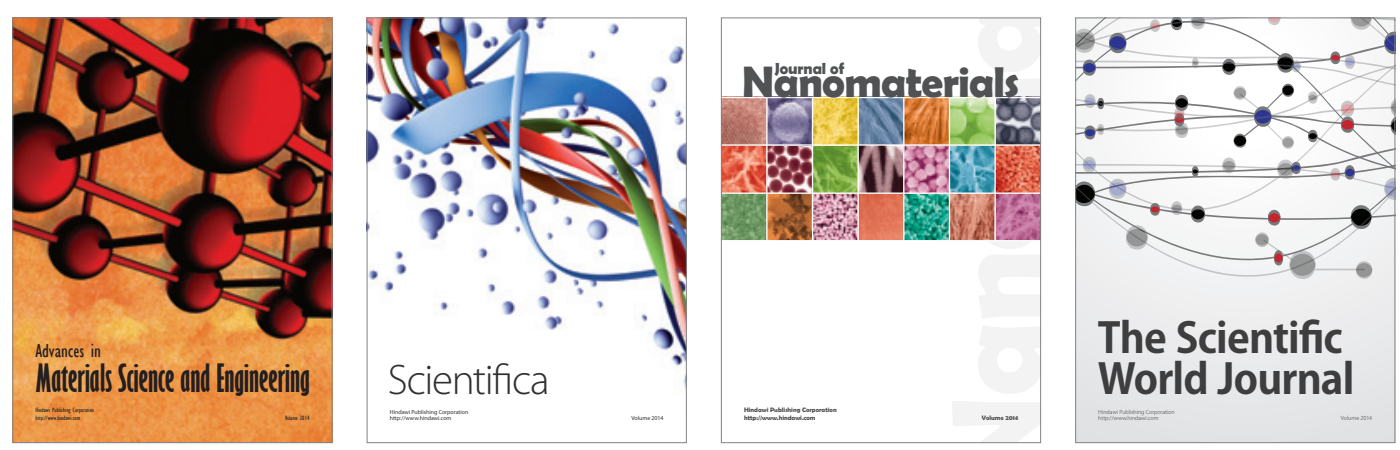

\section{The Scientific World Journal}
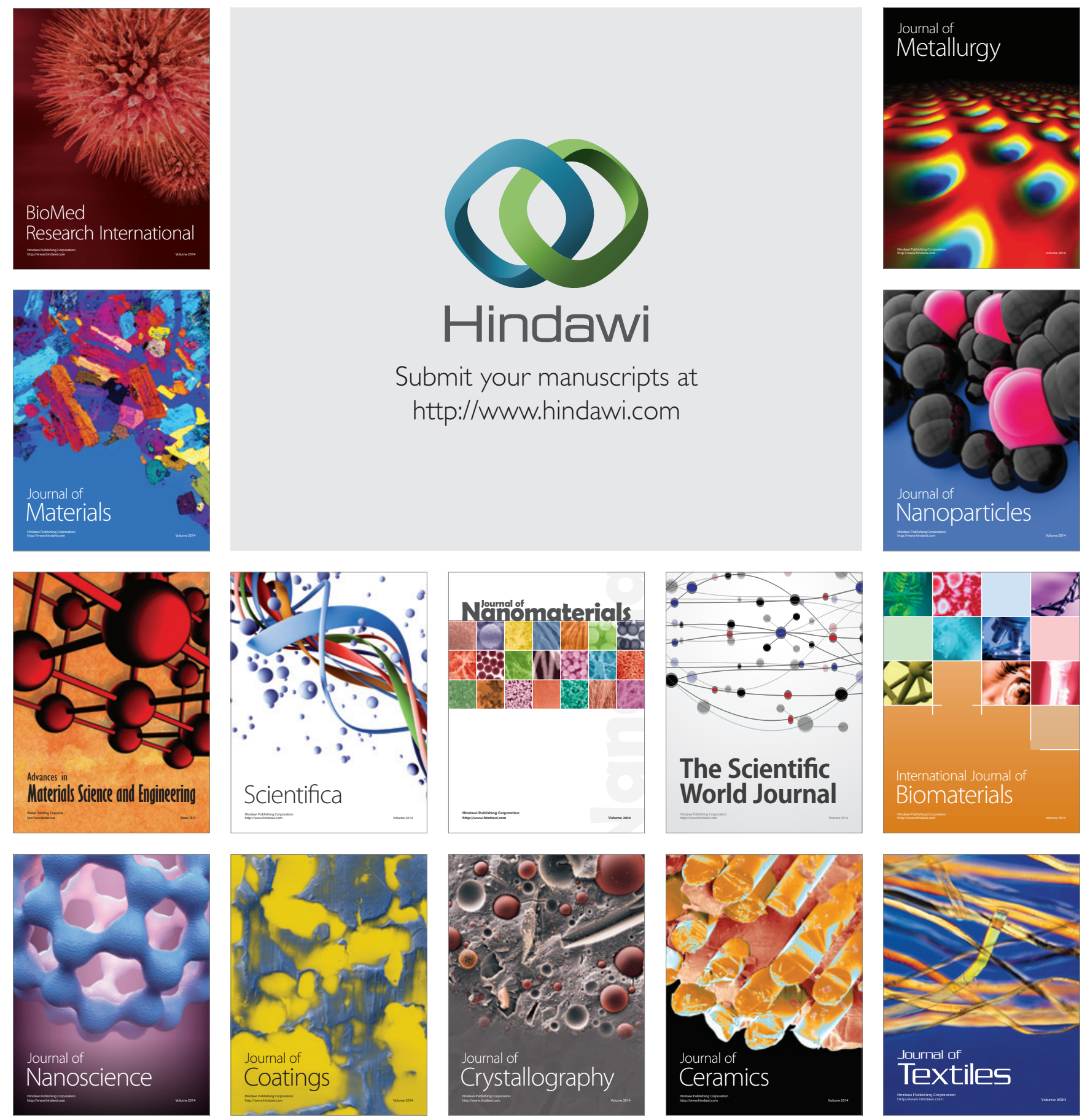